\title{
Identification of SLR Tool Needs - Results of a Community Workshop
}

\author{
Edgar Hassler ${ }^{\mathrm{a}}$, Jeffrey C. Carver ${ }^{\mathrm{b}}$, David Hale ${ }^{\mathrm{a}}$, Ahmed Al-Zubidy ${ }^{\mathrm{b}}$ \\ ${ }^{a}$ Department of Information Systems, Statistics, and Management Sciences, The University of Alabama, Tuscaloosa, AL \\ 35401 \\ ${ }^{b}$ Department of Computer Science, The University of Alabama, Tuscaloosa, AL 35487
}

\begin{abstract}
Context: With the increasing popularity of the Systematic Literature Review (SLR) process, there is also an increasing need for tool support. Objective:The goal of this work was to consult the software engineering researchers who conduct SLRs to identify and prioritize the necessary SLR tool features. Method: To gather information required to address this goal, we invited SLR authors to participate in an interactive 2-hour workshop structured around the Nominal Group Technique. Results: The workshop outcomes indicated that Search \& Selection and Collaboration are the two highest priority tool features. The results also showed that most of the high-priority features are not well-supported in current tools. Conclusion: These results support and extend the results of prior work. SLR tool authors can use these findings to guide future development efforts.
\end{abstract}

Keywords: Systematic Literature Review, Community Workshops

\section{Introduction}

The Systematic Literature Review (SLR) process is a formal, repeatable, documented process for identifying, evaluating, and analyzing the literature relevant to a specific topic or question [1]. With the increase in frequency of various type of empirical studies in software engineering (SE), SLRs are becoming increasingly important as a method to systematically gather and analyze the results from these studies. A 2013 mapping study identified 174 SLRs published in the SE literature [2] between 2005 and 2011. SLRs are valuable to researchers, by providing guidance

Email addresses: ehassler@cba.ua.edu (Edgar Hassler), carver@cs . ua.edu (Jeffrey C. Carver), dhale@ua.edu (David Hale), aalzubidy@crimson.ua.edu (Ahmed Al-Zubidy)

Preprint submitted to Information and Software Technology

October 27, 2015 
about areas in need of further research, and to practitioners, by providing information that can be used in deciding whether or not to adopt a practice within the organization.

Despite these benefits, SLRs are difficult and time consuming to conduct. In most cases, the research team must perform the formal process manually (without adequate tool support). Previously, we conducted a community workshop to identify the key barriers in the SLR process. Participants in that workshop identified the following crucial barriers: 1) lack of tool support for data extraction, 2) low quality of the articles, 3) lack of methods for synthesizing data, 4) inadequate search engines, 5) difficulty in analyzing and presenting qualitative data, and 6) ensuring the SLR topics are relevant to industry [3]. Our belief is that at least four of these six items (i.e. data extraction, data synthesis, inadequate search engines, and analysis/presentation of qualitative data) can be addressed by tool support.

Inadequate tool support increases the effort required for researchers that conduct SLRs and adds barriers for new researchers wishing to perform SLRs. To begin to address these problems, researchers have developed tools to support various aspects of the SLR process. A recent mapping study identified eleven SLR tools in the SE literature [4]. Eight of these tools focus on only one or two aspects of the SLR process: study identification [5], study selection [6, 7, 8, 9], data extraction [8, 10, 11], or data synthesis [10, 12, 13, 14]. The remaining three tools target the entire SLR process [14, 15, 16]. Unfortunately, only two of these tools have been evaluated independently [11]. The lack of integration of tools across the SLR process along with the general lack of external validation suggest the need for additional research to identify the SLR tool features most in need by the community.

Therefore, the goal of this work is to identify and prioritize tool features that would be beneficial when conducting an SLR in software engineering. The results of this work will provide important information to researchers who develop SLR support tools. To address this goal, we enumerate four research questions:

RQ1: Which SLR tool features do SLR authors desire? This basic question is geared towards enumerating all of the important features necessary to address the barriers that SLR authors currently face.

RQ2: What is the development priority for each identified feature? The answer to RQ1 could result in an overwhelming number of desired features. Therefore, to help focus development efforts of tool builders, it is important to prioritize those features. 
RQ3: Does the experience level of the SLR author affect the development priority of the

features? While RQ2 examines the overall priority, it is quite likely that novice SLR authors will have different needs than more experienced SLR authors. This question will help identify those differences and provide additional insight to tool builders about the different audiences.

RQ4: How well do the existing SLR tools support the identified features? Finally, it is important to understand how well the existing SLR tools support these features. If all features are well-supported, then the answer to this question will provide a mapping between requirements and tools. If features are not well-supported, then the answer to this question will provide a roadmap for tool builders about where to focus their efforts to have the most impact.

To answer these questions, an interactive forum like a workshop is preferable to a survey because it allows authors to spend time thinking and discussing these ideas to arrive at the final results. We used the Nominal Group Technique [17] to organize a workshop to elicit the information required to answer the research questions. We conducted this community workshop just prior to the 18th International Conference on Evaluation and Assessment in Software Engineering (EASE'14). We chose this venue because the prevalence of SLRs typically presented at the conference suggested that many SLR authors would already be in attendance.

The primary contributions of this work are:

- A prioritized list of SLR tool features derived from active SE SLR Authors.

- An understanding of the differences in tool feature requirements between novice and experienced SLR Authors.

- An indication the participants' peceptions of which SLR tool features are not met in existing SLR tools.

- A comparison of our results to those of prior studies about SLR tool needs.

Section 2 describes more details about the participants and the workshop methodology. Section 3 reports the outcomes of the workshop along with some analysis of those outcomes. Section 4 discusses the outcomes in relation to extant literature and their implications. Section 5 lists the threats to validity of this study. Section 6 summarizes the paper and describes future work. 


\section{Workshop Methodology}

Consistent with the Research Questions enumerated in Section 1 , the workshop discussion focused on:

- Identifying features that should be provided by SLR-support tools,

- Prioritizing those features, and

- Understanding how well current tools support those features.

Section 2.1 describes the workshop participants. Section 2.2 details the workshop activities.

\subsection{Participants: SLR Authors}

To better understand the tool support desired by SLR authors, we needed to attract participants who were active in conducting SLRs and/or building SLR support tools. We searched the software engineering literature to identify papers reporting SLRs and papers describing SLR support tools. From these papers, we extracted a list of the authors as potential workshop participants. Approximately one month prior to the conference, we invited all of these authors, via email, to participate in the workshop. The invitation informed them that the goal of the workshop was to "gather and prioritize community needs and requirements regarding tool infrastructure to support the SLR process."

Sixteen participants from around the world attended the workshop, including ten SLR experienced authors (i.e. had completed at least three SLRs) and six novice SLR authors (i.e. had completed one or two SLRs). All participants were interested in improving the SLR process by upgrading the available support infrastructure.

\subsection{Workshop Design}

To help the workshop participants effectively interact with each other and to obtain the most accurate information, we used the Nominal Group Technique [17] to organize the workshop activities. The Nominal Group Technique is ideal for workshops with these characteristics [18]:

- Most participants are unfamiliar with each other (i.e. they do not generally work together);

- Some participants may be more vocal than others; 


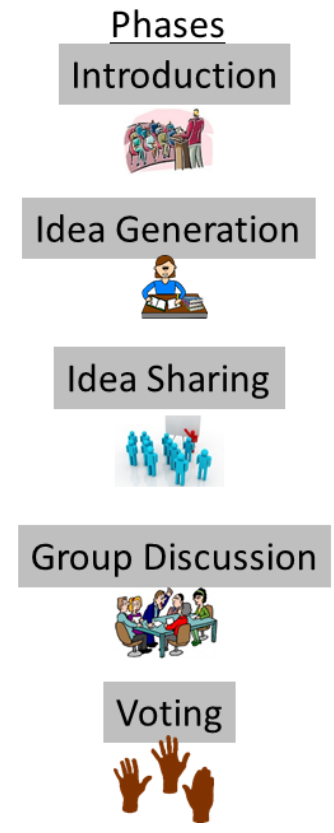

\section{Outputs}
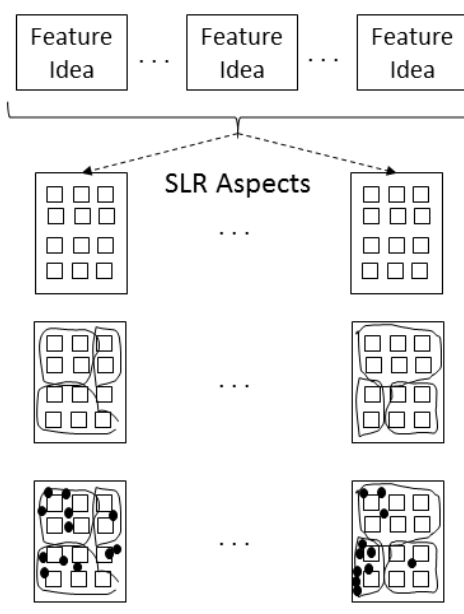

Figure 1: Overview of Methodology

- Some participants think better in silence; and

- Lack of any topics that would be controversial or generate heated interactions.

These characteristics matched our workshop. Figure 1 provides an overview of the five phases of the workshop methodology. The remainder of this section describes these Nominal Group Technique phases in more detail.

\subsubsection{Phase 1: Introduction and Explanation}

We began the workshop with a brief overview of the SLR protocol and process stages to ensure that everyone was using the same terminology and conceptual model. In our previous work [19], we illustrated Kitchenham's SLR process as follows:

- Planning Stage

- Motivation for conducting SLR

- Research questions

- Search strategy (databases and search strings) 
- Strategy for identification of primary studies (inclusion/exclusion criteria)

- Quality assessment criteria

- Data extraction strategy

- Procedure for data synthesis

- Project timeline

- Execution Stage

- Identification of relevant research using the search strategy

- Selection of primary studies using inclusion/exclusion criteria

- Evaluation of primary studies using quality assessment criteria

- Data extraction

- Data synthesis

- Documentation Stage

- Specify the dissemination strategy

- Write the SLR report

- Publish the SLR report.

We then reminded the participants that the goal of the workshop was to identify and prioritize tool features that would be beneficial in conducting an SLR. Finally, we gave the participants an opportunity to ask questions to clarify the procedure and logistics.

\subsubsection{Phase 2: Idea Generation}

To address RQ1, the goal of this phase was for participants to independently brainstorm and record as many ideas as possible related to the topic: tool features that would be beneficial in conducting an SLR. Participants recorded each feature idea on a sticky note along with a brief description of the feature idea.

In addition to the list of feature ideas, to address RQ4, we asked the participants to indicate whether each feature idea already existed in a known tool. To communicate this information, the participants used different color sticky notes to record the feature idea depending upon their knowledge of existing tool support for that idea: 
- Green - the feature idea exists in a known tool and is well done;

- Yellow - the feature idea exists in a known tool, but needs improvement;

- Red - the feature idea does not exist in a known tool.

For any feature idea recorded on a green or yellow sticky note, the participant also had the opportunity to list the tool in which the feature could be found. The participants performed this phase individually and in silence. These choices encouraged participation from everyone and prevented more vocal participants from dominating the discussion. This phase lasted 10 minutes.

\subsubsection{Phase 3: Idea Sharing}

Based on empirical results from previous research [3, 19], we identified six primary aspects of the SLR process for which tool support would be valuable:

- Overall Protocol (i.e., tool feature that is beneficial across two or more SLR stages);

- Motivation for Conducting SLRs;

- Search and Selection;

- Quality Assessment;

- Data Extraction; and

- Analysis and Presentation.

For each of these SLR aspects, we placed a corresponding poster on the wall around the meeting room.

Starting with the Overall Protocol aspect, the participants took turns reading aloud one of his/her feature ideas that related to this SLR aspect. Then the participant affixed the sticky note documenting that feature idea (from the Idea Generation Phase) to the Overall Protocol poster. This process continued until all participants had affixed all of their feature ideas related to Overall Protocol onto the corresponding poster. We then repeated this process for each of the other five SLR aspects.

During this phase, if a participant determined that his or her feature idea fit into multiple SLR aspects, the participant duplicated the sticky note and affixed a copy to the posters corresponding 


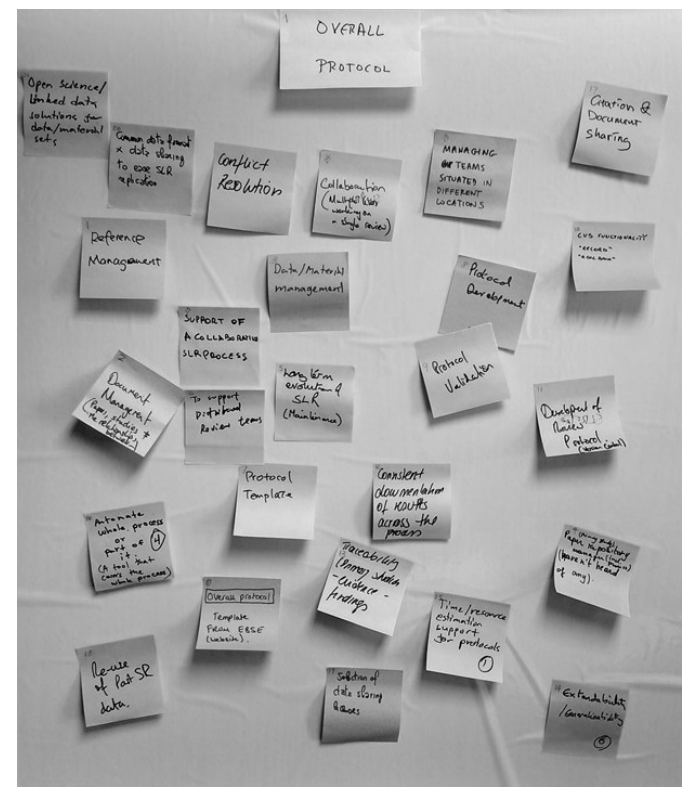

Figure 2: Results of Idea Sharing Phase (full color version: http://carver.cs.ua.edu/Data/EASE_Workshop/ IdeaSharing.jpg)

to each of the relevant SLR aspects. In addition, during this phase, we allowed participants to generate new ideas (i.e. ideas not recorded during the Idea Generation Phase). If a participant identified a new feature idea, s/he recorded the idea on a sticky note (just as in the Idea Generation Phase) and affixed the sticky note to the appropriate poster. This phase lasted approximately 15 minutes. Figure 2 is an example of the poster representing the Overall Protocol aspect at the end of this phase.

\subsubsection{Phase 4: Group Discussion}

After participants had affixed all of their sticky notes to the appropriate poster, the next step was to discuss these feature ideas. This phase consisted of two activities: Concurrent Subgroup Deliberation and Subgroup Presentation and Group Discussion.

Concurrent Subgroup Deliberation. The goal of this activity was to consolidate the feature ideas on the sticky notes into a set of overriding themes, called tool features from now on. We formed four four-person groups and assigned each group to one of the six SLR aspects described in Section 2.2.3 starting with the SLR aspects that contained the most feature ideas. When a group 


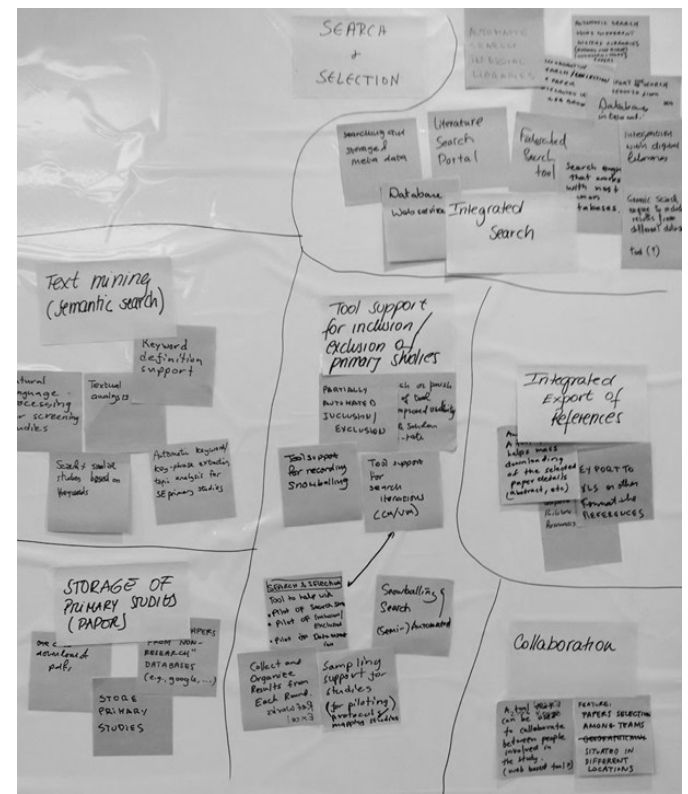

Figure 3: Results of Group Discussion Phase (full color version: http://carver.cs.ua.edu/Data/EASE_ Workshop/GroupDiscussion.jpg)

finished their assigned SLR aspect, we asked them to work on one of the remaining SLR aspects.

The participants began by examining all of the sticky notes on their assigned SLR aspect poster and grouping together related feature ideas. Sometimes these groupings had many similar (or identical) feature ideas from multiple sticky notes, while other times a sticky note contained a unique feature idea that could not be grouped with any other ones. During their discussion, if group members thought any of the feature ideas assigned to their SLR aspect fit better in another SLR aspect, they could offer that feature idea to the group discussing that SLR aspect. Therefore, groups could move feature ideas around to arrive at the most optimal allocation among the SLR aspects. This activity resulted in a set of tool features associated with each SLR aspect. We gave the participants 30 minutes to complete this activity during which we were available to answer questions and provide clarifications. Figure 3 is an example of the poster representing the Search \& Selection aspect the participants grouped the feature ideas into tool features.

Subgroup Presentation and Group Discussion. The goal of this activity was to allow all participants to comment on the set of tool features identified for each SLR aspect. A presenter from each SLR aspect described the identified tool features. After the presentations, the participants 
discussed the relationships among the tool features within each SLR aspect and across SLR aspects.

\subsubsection{Phase 5: Voting and Ranking}

After there was agreement on the identified tool features, to address RQ2, the participants prioritized those tool features. Considering all of the tool features across all SLR aspects the participants decided which ones were most important, i.e. had the highest priority for being addressed. To record the voting, we gave each participant nine dots, which represented $33 \%$ of the total number of tool features identified in the Group Discussion Phase. To gather data for RQ3, we gave experienced SLR authors different color voting dots than novice SLR authors. To prevent any bias, we did not inform the participants of the meaning of the different colored dots.

Because the total number of votes given to each tool feature is the indication of its relative priority, each participant could assign his/her nine votes in any manner s/he chose. For example, if a participant though one tool feature was the most important and the only one worth considering, s/he could assign all nine votes to that tool feature. Conversely, if another participant considered nine tool features to be of equal importance, s/he could assign one vote to each of those nine tool features. At the end of this phase, we had the ordered priority of the tool features. Figure 4 is an example of the Search $\&$ Selection poster after the voting phase.

\section{Results}

This section reports the results generated during the workshop. It primarily focuses on counting and tabulation of the votes cast along with some general observations. Section 4 discusses the implications of these results.

\subsection{Overall Results}

During the Idea Generation Phase (Section 2.2.2], the participants recorded 91 unique feature ideas that should be supported by SLR tools. Then, during the Idea Sharing Phase (Section 2.2.3, the participants identified 6 additional feature ideas. Finally, during the Group Discussion Phase (Section 2.2.4, the groups moved 6 feature ideas from one aspect to another. Table 1 summarizes the distribution of the 97 feature ideas across the six SLR aspects after the Group Discussion Phase. 


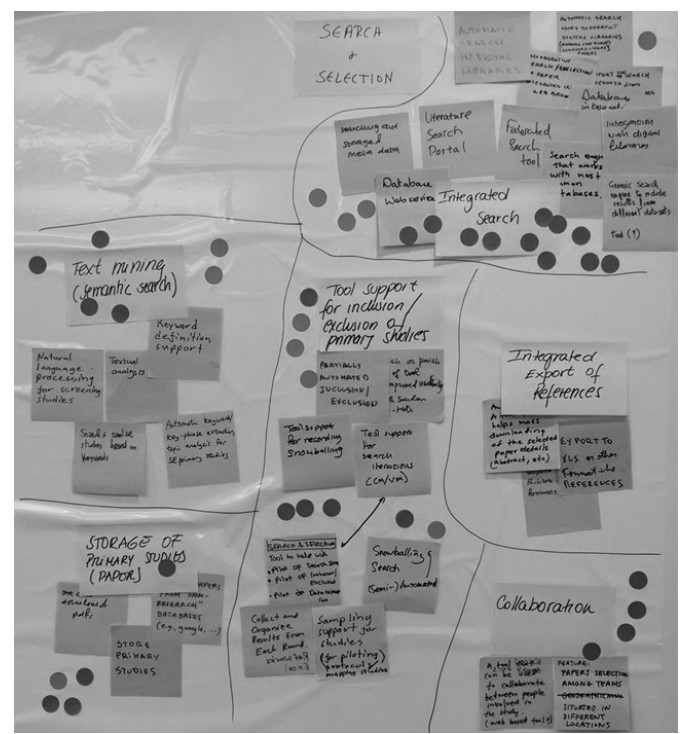

Figure 4: Results of Voting Phase (full color version: http://carver.cs.ua.edu/Data/EASE_Workshop/Voting. jpg)

Table 1: Overall Results

\begin{tabular}{c|c} 
SLR Aspects & Feature Ideas \\
\hline Overall Protocol (OP) & 27 \\
Motivation \& Research Questions (MR) & 1 \\
Search \& Selection (SS) & 33 \\
Quality Assessment (QA) & 4 \\
Data Extraction (DE) & 12 \\
Analysis \& Presentation (AP) & 20 \\
\hline
\end{tabular}




\subsection{Research Questions}

The following subsections present the data relevant to each research question.

\subsubsection{RQ1: Which SLR tool features do SLR authors desire?}

The participants consolidated the 97 feature ideas into 28 tool features. Table 2 presents the results of the end of the Group Discussion Phase grouped by SLR Aspect. As shown in the table, some tool features resulted from the grouping of a large number of feature ideas (maximum of 12). Conversely, other features ideas were unique and deemed by the sub-group to be important enough on their own to be a tool feature. Note that the number of feature ideas consolidated into a tool feature should not be considered to be a proxy for the value of that tool feature. The results from Research Questions 2 and 3 investigate the relative value of the features in more detail.

\subsubsection{RQ2: What is the development priority for each identified feature?}

Table 3 lists the tool features (prefaced by the 2-letter code representing the corresponding SLR aspect) in order of priority based on the results of the Voting and Ranking Phase. For these analyses, we converted the raw number of votes into percentages to make them easier to understand. That is, we divided the number of votes received by each tool feature by 144 (16 participants $* 9$ votes). We can make a few interesting observations from this data.

- The highest priority tool feature is integrated search, which is the ability to search multiple databases without having to perform separate searches.

- The top five highest-priority tool features come from the Overall Protocol and Search $\boldsymbol{\&}$ Selection aspects of the SLR process.

- The concept of collaboration appears in four tool features $(O P$ - Collaboration through the SLR process, SS - Collaboration, DE - Collaboration support, and AP - Collaboration) across four SLR aspects. In total, these four features received $16.9 \%$ of the votes, giving Collaboration the highest priority overall.

- Several of the tool features refer to task-level actions within the Searching and Selection and Analysis \& Presentation SLR aspects.

- Five of the tool features received no votes. 
Table 2: Results of Group Discussion Phase

\begin{tabular}{|c|c|}
\hline SLR Aspect (6) & SLR Tool Feature (28) \\
\hline Overall Protocol (OP) & $\begin{array}{c}\text { Collaboration } \\
\text { Data sharing } \\
\text { Data maintenance } \\
\text { Traceability } \\
\text { Development \& validation } \\
\text { Automate tasks } \\
\text { Resource estimation \& management } \\
\text { Extendability }\end{array}$ \\
\hline Motivation \& Research Questions (MR) & Templating \\
\hline Search \& Selection (SS) & $\begin{array}{c}\text { Integrated search } \\
\text { Study selection } \\
\text { Semantic search } \\
\text { Studies storage } \\
\text { Export of references } \\
\text { Collaboration }\end{array}$ \\
\hline Quality Assessment (QA) & $\begin{array}{c}\text { Process standardization } \\
\text { Quality assessment }\end{array}$ \\
\hline Data Extraction (DE) & $\begin{array}{c}\text { Coding: Methods \& data } \\
\text { Technical mgmt } \\
\text { Collaboration } \\
\text { Piloting }\end{array}$ \\
\hline Analysis \& Presentation (AP) & $\begin{array}{c}\text { Automated analysis } \\
\text { Statistical analysis } \\
\text { Visualization } \\
\text { Validation } \\
\text { Extendability } \\
\text { Collaboration } \\
\text { Index creation }\end{array}$ \\
\hline
\end{tabular}


Table 3: Results of Voting Phase

\begin{tabular}{l|c} 
Feature & Votes \\
\hline SS - Integrated search & $11.5 \%$ \\
OP - Collaboration & $10.7 \%$ \\
OP - Traceability & $9.2 \%$ \\
SS - Study selection & $6.9 \%$ \\
OP - Data maintenance & $6.1 \%$ \\
QA - Quality assessment & $5.3 \%$ \\
AP - Automated analysis & $5.3 \%$ \\
SS - Semantic search & $4.6 \%$ \\
SS - Studies storage & $3.8 \%$ \\
DE - Coding: Methods \& data & $3.8 \%$ \\
AP - Visualization & $3.8 \%$ \\
OP - Automate tasks & $3.1 \%$ \\
SS - Collaboration & $3.1 \%$ \\
DE - Technical mgmt & $3.1 \%$ \\
DE - Collaboration & $3.1 \%$ \\
QA - Process standardization & $3.1 \%$ \\
AP - Validation & $3.1 \%$ \\
OP - Data sharing & $2.3 \%$ \\
AP - Statistical analysis & $2.3 \%$ \\
MR - Templating & $2.3 \%$ \\
AP - Extendability & $2.2 \%$ \\
OP - Development \& validation & $0.8 \%$ \\
OP - Resource estimation \& management & $0.8 \%$ \\
OP - Extendability & $0.0 \%$ \\
SS - Export of references & $0.0 \%$ \\
DE - Piloting & $0.0 \%$ \\
AP - Collaboration & $0.0 \%$ \\
AP - Index creation & $0.0 \%$ \\
\hline
\end{tabular}


3.2.3. RQ3: Does the experience level of the SLR author affect the development priority of the features?

Tables 4 and 5 show the tool features with the largest differences in priority between the experienced and the novice SLR authors, respectively. We can make some observations about the differences in priority given to the features by these two groups. Based on votes cast:

- There is no overlap in top valued new tool features between the Novice- and High-Experienced SLR authors

- The experienced SLR authors identified Support for Collaboration (in the Overall Protocol and Search and Selection Aspects) with a much higher priority than the novice SLR authors ( $17.7 \%$ to $4.9 \%$ respectively); and

- The highest priority feature overall, Integrated Search, was not the highest priority feature for either the experienced or the novice SLR authors.

Thus, as a generalization,

- The Novice SLR authors cast their votes much more often than the High-Experienced SLR authors for new tool features that aid in executing primary operational tasks such as analyzing, tracing, time estimating, and collaboration on data extraction.

- The High-Experienced SLR authors cast their votes much more often than the Novice SLR authors for new tool features that aid in tactical activities such as collaboration, protocol development and validation, and sharing.

\subsubsection{RQ4: How well do the existing SLR tools support the identified features?}

Table 6 shows the perceived level of tool support for the eight highest-priority features (based on Table 3). This data came from the Idea Generation Phase, where participants recorded desired feature ideas on different color sticky notes depending on their perception of how well existing tools supported that feature idea. Note that because tool features may consist of different numbers of feature ideas, the total number of red, yellow, and green sticky notes differs (i.e. a feature consisting of 12 feature ideas will have 12 sticky notes, while a feature consisting of only 1 idea will have only 1 sticky note). Therefore, the raw numbers in Table 6 are not as important as the overall trend. 
Table 4: Highest Priority SLR Tool Features (Experienced SLR Authors)

\begin{tabular}{l|c|c|c}
\multirow{2}{*}{ Tool Feature } & \multicolumn{2}{|c|}{ Votes } & Difference \\
\hline OP - Collaboration & $13.3 \%$ & $4.9 \%$ & $8.5 \%$ \\
QA - Quality assessment & $6.7 \%$ & $2.4 \%$ & $4.2 \%$ \\
SS - Study storage & $4.4 \%$ & $2.4 \%$ & $2.0 \%$ \\
SS - Collaboration & $4.4 \%$ & $0 \%$ & $4.4 \%$ \\
OP - Data sharing & $3.3 \%$ & $0 \%$ & $3.3 \%$ \\
OP - Development \& validation & $1.1 \%$ & $0 \%$ & $1.1 \%$ \\
\hline
\end{tabular}

Table 5: Highest Priority SLR Tool Features (Novice SLR Authors)

\begin{tabular}{l|c|c|c}
\multirow{2}{*}{ Tool Feature } & \multicolumn{2}{|c|}{ Votes } & Difference \\
& Novice & Experienced & \\
\hline AP - Automated analysis & $9.8 \%$ & $3.3 \%$ & $6.4 \%$ \\
OP - Traceability & $12.2 \%$ & $7.8 \%$ & $4.4 \%$ \\
SS - Study selection & $9.8 \%$ & $5.6 \%$ & $4.2 \%$ \\
AP - Statistical analysis & $4.9 \%$ & $1.1 \%$ & $3.8 \%$ \\
DE - Collaboration & $4.9 \%$ & $2.2 \%$ & $2.7 \%$ \\
OP - Resource estimation \& management & $2.4 \%$ & $0 \%$ & $2.4 \%$ \\
\hline
\end{tabular}




\begin{tabular}{l|c|c|c|c|c|c}
\multicolumn{7}{|c}{ Table 6: Perceived Tool Support For High Priority Features } \\
Aspect - Tool Feature & Missing & \multicolumn{2}{|c|}{ Needs Improvement } & \multicolumn{2}{c}{ Well Done } \\
& & Votes & Tools & Votes & Tools \\
\hline SS - Integrated search & 6 & 6 & SLuRP [15] & 0 & None \\
OP - Collaboration & 4 & 1 & None & 1 & SLuRP [15] \\
OP - Traceability & 2 & 0 & N/A & 2 & SLRTOOL [20] \\
SS - Study selection & 5 & 3 & Excel & 0 & N/A \\
OP - Data maintenance & 2 & 2 & None & 1 & None \\
QA - Quality assessment & 1 & 0 & N/A & 0 & N/A \\
AP - Automated analysis & 3 & 4 & None & 0 & N/A \\
SS - Semantic search & 3 & 2 & None & 0 & N/A \\
\hline \multicolumn{2}{c}{ Note that in each case, each tool was mentioned by only one participant. }
\end{tabular}

During the Idea Generation Phase, most participants did not identify which tool(s) fully or partially supported the features listed. Table 6 lists the tools identified for the eight highest priority features. In each case, the tool was only mentioned by one participant. In addition to those features, the participants also identified SLuRP [15] as having support for the following features: $O P$ - Protocol Development (needs improvement), DE - Collaboration (needs improvement), DE - Coding (needs improvement), AP - Extendability (well done), and AP - Collaboration (well done).

We note the following observations about the participants' perception of the current level of tool support.

- The two highest-priority tool features, Integrated Search and Collaboration, are not wellsupported by current tools.

- The third highest-priority tool feature, Traceability, has a dichotomous result. Two feature ideas currently do not exist in SLR authoring tools. Two feature ideas are well done in current tools.

- The remaining five highest-priority tool features were either missing or needed improvement, with the exception of Data Maintenance (indicated by one participant as being well done). 


\section{Discussion}

Subsequent to our workshop, Marshall and his colleagues published two papers related to SLR tooling. In the first paper, Marshall et al. use their own experiences, a preliminary screening of candidate tools, and discussions among the authors to enumerate a set of SLR tool features that would improve the SLR process [21]. In the second paper, Marshall et al. use the results of interviews of SLR authors from multiple domains to understand the desirability of SLR tool features [22]. This section compares the results of our workshop to these previous papers to highlight common results (Section 4.1) and to identify new findings about the needs for SLR tool support (Section 4.2). Table 7 maps our results to those from Marshall et al.'s studies. For our work, the table shows the SLR aspects along with the percentage of votes cast as an indicator of relative priority. For Marshall et al.'s work, the table shows whether the identified feature was Mandatory, Highly Desirable, Desirable, Nice-to-Have, or Not Necessary in each of the two studies.

\subsection{Common Results}

A common theme that consistently appears across all three studies is the need for an integrated tool set. When we combine the results of all three studies, we obtain a clearer view of the meaning of an integrated tool set. An integrated tool set must provide:

- Collaboration among the SLR team, including role management, security, dispute resolution and coordination;

- Automation of the processes and tasks;

- Data sharing between processes and tasks, and among the SLR team;

- Data maintenance and preservation functions to access past (and support future) research questions, protocols, studies, data, meta-data, bibliographic data and reports; and

- Forward and backward traceability to link goals, actions, and results for accountability, standardization, verification and validation.

Collectively, integration is essential across the SLR tasks from the projects inception to protocol execution and dissemination of the final report. 


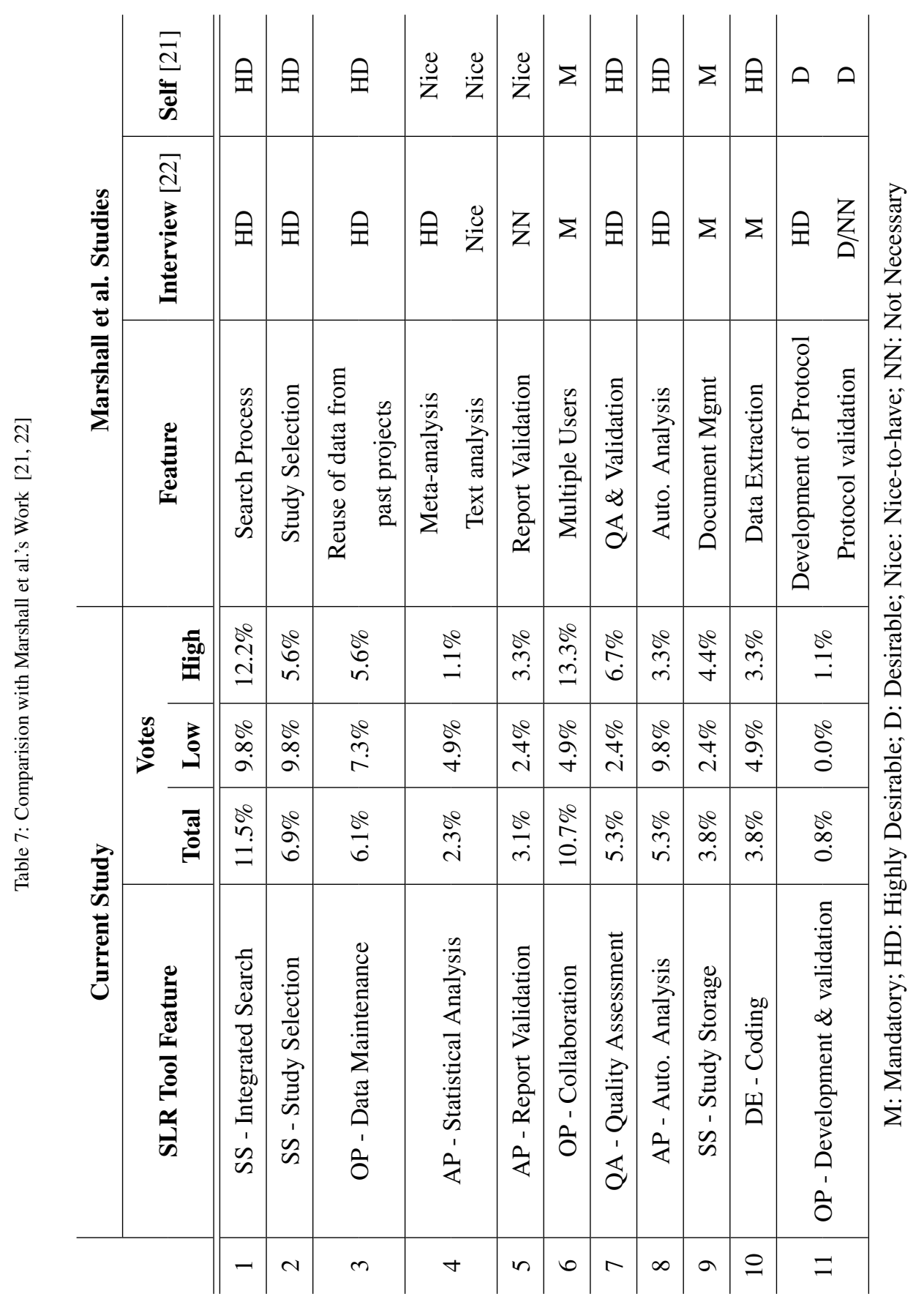


Beyond the characterization of an integrated tool, we found several tool features in common between the studies:

- Tool features 1 and 2 SS-Integrated Search, and SS-Study Selection, respectively were of high importance in each of the studies.

- Tool feature 3 OP-Data Maintenance was of high importance in our study and the Marshall et al. [2015] study.

- Tool feature 4 AP-Statistical Analysis was of moderate importance in our study and nice to have in the Marshall et al studies.

- Tool feature 5 AP-Report Validation was of lower importance in our study and was found to be Not Applicable or a Nice-to-Have in the Marshall et al. studies.

- Tool features 6 and 7 OP-Collaboration and QA-Quality Assessment were mandatory and highly desirable (respectively) in the Marshall et al. studies and by experienced authors in our study. However, as mentioned in Section 3 , novice SLR authors did not place a high priority on these two features.

- Tool feature 8 AP-Automated Analysis was desirable in both of the Marshall et al. studies and among novice SLR authors in our study, but not by the experienced SLR authors in our study.

- Tool features 9 and 10 SS-Studies Storage and DE-Coding: Methods $\mathcal{F}$ Data were mandatory or highly desirable in the Marshall et al. studies. In our study both features had moderate prioritization, being ranked 9 th and 10th overall in the voting.

\subsection{Difference in Results}

Due to the mechanism used to collect the authors prioritization in our study Tool Feature 11 OP-Development $\mathcal{E}$ Validation maps to two features defined by Marshall et al. Development of review protocol and Protocol Validation. The Marshall et al. studies found these features to range from highly desirable to desirable to not necessary. To further confound this feature, the OP-Traceability in our study seems similar but was not explicitly identified in the Marshall et al. studies. 
The Marshall et al. studies included administrative, operational, financial, and systems architecture tool requirements that go beyond the functional execution we focused on in our study. These requirements include: low or no cost, software maintenance, simple installation and setup procedures, software install guide, and tutorials. Though the participants in our study did not provide input into these requirements, we concur these are important considerations and need to be addressed.

In addition, our study identified some tool features that the Marshall et al. studies did not explicitly identify.

- Semantic search: our results also indicate that toolsmiths should employ outside-the-box thinking with regard to automating portions of the process. Most literature searches are currently conducted based on keywords in combination with standard Boolean operators. However, a high ranking feature requested by the workshop participants is the ability to conduct a semantic search of the literature that is akin to text mining. Such innovations will not only reduce the time required to complete a SLR, but also assist with the cognitive load encountered in translating the search strategy into executable queries.

- Visualization of Extracted and Analyzed Data: discussion among the SLR authors revealed the desire to have tool support for data visualization. The visualization mechanisms can support selection, analysis, synthesis, and conveyance of results.

- Piloting of the data extraction process: as Kitchenham and Charters [1] suggest, piloting of the entire protocol is important. One can extrapolate this feature to the other tasks of the SLR process. Additionally, novice SLR authors indicated a need for tools supporting the estimation of time and resources needed to conduct a SLR.

- Time/Resource estimation/management: from a project management standpoint, metadata concerning the pilot effort could be utilized to estimate or refine projections of time and resource requirements.

\section{Threats to Validity}

In conducting this workshop, we identified some threats to validity that may reduce the confidence a reader could place in the results or the overall generalizablity of the results. 
Internal Validity. The main threat to interal validity is related to the two-hour time-limit of the workshop. This time limit might have constrained both the idea generation and group discussions of the participants. Given additional time, participants may have identified additional tool features. Based on the outcomes of the workshop, we do not consider this threat serious as most participants completed the idea generation and group discussion phases within the allocated time.

Construct Validity. The main threat to construct validity was our use of participant experience as a proxy for SLR proficiency. In addition, we chose a threshold of three SLRs to differentiate between novice and experienced SLR authors. It is possible that the results could have changed if we used a different measure of proficiency or a different threshold to separate novice from expert. At this time, we did not have another feasible method to measure proficiency. More study is needed to determine whether there is a better way to measure proficiency in conducting SLRs.

External Validity. The main threat to external validity was the sample of workshop attendees. Even though we invited all SLR authors that we identified from our literature search, the actual participants were self-selected. The ability, or lack thereof, to travel to the workshop potentially resulted in a non-representative sample. We have no evidence that our sample was biased. In fact, the sample contained participants from around the world and with different levels of experience. Therefore, to the degree that the workshop participants represent SLR authors as a whole the result present here may be generalized.

\section{Summary}

This paper describes the results of a community workshop geared towards identifying and prioritizing SLR tool requirements. Our findings provide guidance to SLR tool developers regarding priorities as perceived by current SLR authors. The key results from this work include:

- Search $\mathcal{E}$ Selection and Collaboration are the most desired SLR tool features.

- The relative priorities of the identified tool features differs between novice and experienced SLR authors.

- The workshop participants perception was that current tools do not fully support the most important SLR tool features. 
- Our results were consistent with those of similar studies, but also provided some additional insight.

Our future work is to validate the conclusion that current tools lack support for the key tool features identified in our work. To perform this validation, we will manually examine the existing tools and interact with the tool developers. For tool features that are not well-supported by current SLR tools, we will enumerate the tool requirements in more detail and begin implementing these features in our own tools.

\section{Acknowledgments}

This material is based upon work supported by the U.S. National Science Foundation under Grant Number 1305395. The authors are grateful to all involved in these studies, particularly the workshop participants for their insights and cooperation, and the EASE 2014 organizers for allowing us to conduct the workshop.

\section{References}

[1] B. Kitchenham, S. Charters, Guidelines for performing systematic literature reviews in software engineering, Joint Report EBSE 2007-001, Keele University and Durham University (2007).

[2] S. Imtiaz, M. Bano, N. Ikram, M. Niazi, A tertiary study: Experiences of conducting systematic literature reviews in software engineering in: Proceedings of the 17th International Conference on Evaluation and Assessment in Software Engineering, EASE '13, ACM, New York, NY, USA, 2013, pp. 177-182. doi:10.1145/2460999. 2461025

URL http://doi.acm.org/10.1145/2460999.2461025

[3] E. Hassler, J. C. Carver, N. A. Kraft, D. Hale, Outcomes of a community workshop to identify and rank barriers to the systematic literature review process, in: Proceedings of the 18th International Conference on Evaluation and Assessment in Software Engineering, 2014, pp. 267-276.

[4] C. Marshall, P. Brereton, Tools to support systematic literature reviews in software engineering: A mapping study, in: Empirical Software Engineering and Measurement, 2013 ACM / IEEE International Symposium on, 2013, pp. 296-299. doi:10.1109/esem.2013.32

[5] M. Ghafari, M. Saleh, T. Ebrahimi, A federated search approach to facilitate systematic literature review in software engineering, International journal of software engineering \& applications.

[6] K. R. Felizardo, G. F. Andery, F. V. Paulovich, R. Minghim, J. C. Maldonado, A visual analysis approach to validate the selection review of primary studies in systematic reviews, Information and Software Technology 54 (10) (2012) 1079-1091. doi:http://dx.doi.org/10.1016/j.infsof.2012.04.003 
[7] K. Felizardo, N. Salleh, R. Martins, E. Mendes, S. MacDonell, J. Maldonado, Using visual text mining to support the study selection activity in systematic literature reviews, in: 2011 International Symposium on Empirical Software Engineering and Measurement, 2011, pp. 77-86.

[8] Y. Sun, Y. Yang, H. Zhang, W. Zhang, Q. Wang, Towards evidence-based ontology for supporting systematic literature review, in: 16th International Conference on Evaluation Assessment in Software Engineering, 2012, pp. $171-175$.

[9] F. Tomassetti, G. Rizzo, A. Vetro, L. Ardito, M. Torchiano, M. Morisio, Linked data approach for selection process automation in systematic reviews, in: 15th Annual Conference on Evaluation and Assessment in Software Engineering, 2011, pp. 31-35. doi:10.1049/ic.2011.0004

[10] K. R. Felizardo, E. Nakawaga, D. Feitosa, R. Minghim, J. C. Maldonado, An approach based on visual text mining to support categorization and classification in the systematic mapping, in: Proc. of EASE, Vol. 10, 2010, pp. 1-10.

[11] J. A. S. Torres, D. S. Cruzes, L. do Nascimento Salvador, Automatic results identification in software engineering papers. is it possible?, in: 12th International Conference on Computational Science and Its Applications, 2012, pp. 108-112. doi:10.1109/iccsa.2012.27

[12] D. Cruzes, M. Mendonca, V. Basili, F. Shull, M. Jino, Automated information extraction from empirical software engineering literature: Is that possible?, in: Empirical Software Engineering and Measurement, 2007. ESEM 2007. First International Symposium on, 2007, pp. 491-493. doi:10.1109/esem.2007.62

[13] D. Cruzes, M. Mendona, V. Basili, F. Shull, M. Jino, Using context distance measurement to analyze results across studies, in: Empirical Software Engineering and Measurement, 2007. ESEM 2007. First International Symposium on, IEEE, 2007, pp. 235-244

[14] K. R. Felizardo, M. Riaz, M. Sulayman, E. Mendes, S. G. MacDonell, J. C. Maldonado, Analysing the use of graphs to represent the results of systematic reviews in software engineering, in: 25th Brazilian Symposium on Software Engineering, 2011, pp. 174-183. doi:10.1109/sbes.2011.9

[15] D. Bowes, T. Hall, S. Beecham, Slurp - a tool to help large complex systematic literature reviews deliver valid and rigorous results, in: 2nd International Workshop on Evidential Assessment of Software Technologies, Proceedings of the 2nd International Workshop on Evidential Assessment of Software Technologies, Association for Computing Machinery, 2012, pp. 33-36. doi:10.1145/2372233.2372243

[16] A. M. Fernandez-Saez, M. G. Bocco, F. P. Romero, Slr-tool: A tool for performing systematic literature reviews, in: 5th International Conference on Software and Data Technologies, Vol. vol.2 of Proceedings of 5th International Conference on Software and Data Technologies, INSTICC Press, 2010, pp. 157-66.

[17] A. Delbecq, A. Van de Ven, D. Gustafson, Group techniques for program planning: a guide to nominal group and Delphi processes. Management applications series, Scott, Foresman, 1975 URL https://books .google.com/books?id=1jhHAAAAMAAJ

[18] T. C. Lethbridge, S. E. Sim, J. Singer, Studying software engineers: Data collection techniques for software field studies, Empirical Software Engineering 10 (3) (2005) 311-341.

[19] J. C. Carver, E. Hassler, E. Hernandes, N. A. Kraft, Identifying barriers to the systematic literature review process, in: Empirical Software Engineering and Measurement, 2013 ACM / IEEE International Symposium on, 2013, pp. 203-212. doi:10.1109/esem.2013.28

[20] B. S. Barn, F. Raimondi, L. Athappian, T. Clark, Slrtool: A tool to support collaborative systematic literature 
reviews, in: ICEIS 2014 - Proceedings of the 16th International Conference on Enterprise Information Systems, Volume 2, Lisbon, Portugal, 27-30 April, 2014, 2014, pp. 440-447. doi:10.5220/0004972204400447

[21] C. Marshall, P. Brereton, B. Kitchenham, Tools to support systematic reviews in software engineering: A feature analysis, in: Proceedings of the 18th International Conference on Evaluation and Assessment in Software Engineering, 2014, pp. 13:1-13:10.

[22] C. Marshall, P. Brereton, B. Kitchenham, Tools to support systematic reviews in software engineering: A crossdomain survey using semi-structured interviews, in: Proceedings of the 19th International Conference on Evaluation and Assessment in Software Engineering, 2015, pp. 26:1-26:6. 